\title{
Alimentación del gobio (Gobio gobio (L., 1758)) en el río Larraun (Navarra, N. España).
}

\author{
Oscoz, J., F. Campos y M. C. Escala. \\ Dpto. Zoología y Ecología, Facultad de Ciencias, Universidad de Navarra, Apdo. 177, 31080 Pamplona. \\ E-mail: joscoz@alumni.unav.es
}

\begin{abstract}
RESUMEN
En el río Larraun (norte de España) el gobio (Gobio gobio) se alimentó de invertebrados bentónicos, invertebrados terrestres y materia vegetal. Las presas más consumidas fueron quironómidos, cladóceros y larvas de tricópteros, pero con ciertas diferencias en las abundancias de estos grupos en los dos puntos estudiados. También hubo diferencias entre la dieta de los gobios de edad $\leq 2$ y los de edad $>2$. Los gobios seleccionaron negativamente ácaros, gammáridos, élmidos, efémeras y plecópteros, mientras quironómidos, limónidos y psicómidos fueron seleccionados positivamente.
\end{abstract}

Palabras clave: Gobio, Gobio gobio, alimentación, España.

\begin{abstract}
The gudgeon (Gobio gobio) in Larraun river (North of Spain) was found to feed mainly on benthic macroinvertebrates (Chironomidae, Anomopoda and Trichoptera larvae), although terrestrial invertebrates and plant material were also ingested. There were differences in the feeding habits of gudgeon along the Larraun river, as well as differences between ages classes $\leq 2$ and $>2$. Gudgeon rejected certain prey types (Hidracarina, Gammaridae, Elmidae, Ephemeroptera and Plecoptera), while showing preference for other groups (Chironomidae, Limoniidae and Psychomyidae).
\end{abstract}

Keywords: Gudgeon, Gobio gobio, food, Spain.

\section{INTRODUCCIÓN}

El gobio (Gobio gobio) es una especie paleártica ampliamente distribuida por Europa (Mann, 1980) y Asia central. Durante años se ha considerado a esta especie como introducida en la Península Ibérica (Doadrio y Elvira, 1986), aunque en la actualidad se le considera autóctona en las cuencas de los ríos Ebro y Bidasoa (Doadrio, 2001).

La biología de esta especie en Europa ha sido estudiada por varios autores (Kennedy \& Fitzmaurize, 1972; Mann, 1980; Neveu 1981; Chappaz et al., 1988). Los trabajos realizados en la Península Ibérica se han centrado principalmente en su crecimiento y estrategia reproductora (Lobón-Cerviá y Torres, 1983; Lobón-Cerviá et al., 1991), pero muy poco se ha estudiado sobre su dieta: sólo existe un trabajo realizado en el río Sorraia, Portugal (Magalhães, 1993). Teniendo en cuenta que este tipo de estudios son una importante herramienta de cara a la gestión de las poblaciones piscícolas, con el presente trabajo se quiere dar a conocer mejor las preferencias tróficas de esta especie. De este modo se podrá valorar la posible competencia trófica entre el gobio y las especies nativas en aquellas cuencas donde ha sido introducido.

\section{ÁREA DE ESTUDIO}

Este trabajo se ha realizado en el río Larraun, norte de España. Se trata de un río corto $(21 \mathrm{~km}$ de recorrido y $221 \mathrm{~km}^{2}$ de cuenca) que nace a 650 m.s.n.m. en la Sierra de Aralar, desembocando en el río Arakil. Discurre en su mayor parte por un sustrato calizo, con una pendiente 
media del 1,02\%. El lecho del río está compuesto por losas, bloques y cantos, mientras que la vegetación de ribera está constituida principalmente por alisos (Alnus glutinosa), sauces (Salix sp.), chopos (Populus sp.) y diferentes especies del género Quercus. Además del gobio, otras especies piscícolas presentes en el río Larraun son trucha común (Salmo trutta), piscardo (Phoxinus phoxinus), madrilla (Chondrostoma miegii), barbo de Graells (Barbus graellsii), locha (Barbatula barbatula) y trucha arco iris (Oncorhynchus mykiss), esta última procedente de una piscifactoría.

El estudio se realizó en dos puntos (P1 y P2) de la parte baja del río Larraun (Fig. 1), ya que es en este tramo donde se encontraba la mayor parte de la población de gobios. El punto P1 estaba situado aguas abajo de una presa, siendo

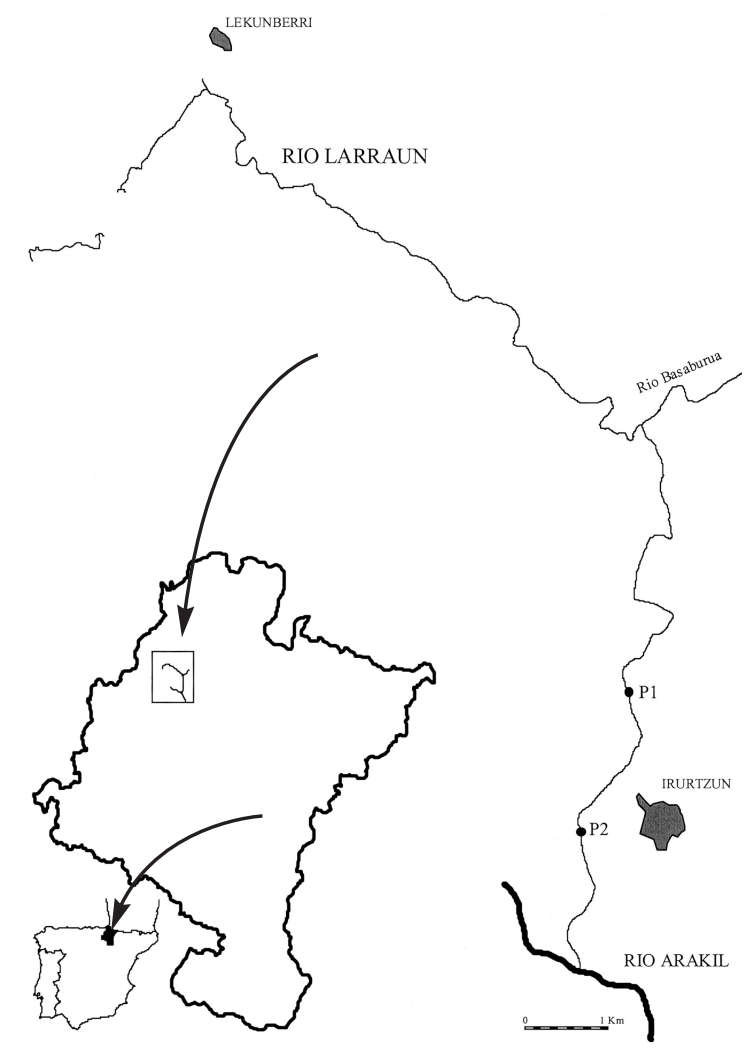

Figura 1. Localización de los puntos de muestreo (P1 y P2) en el río Larraun. Location of the sampling sites (P1 and P2) in the Larraun River (Navarra, North of Spain). una zona de sustrato uniforme y remansada, con una profundidad homogénea (media de 57 $\mathrm{cm}$ ) y una anchura media de $22.3 \mathrm{~m}$. El punto P2 combinaba zonas remansadas y de corriente, teniendo también un mayor rango de profundidades (media de $42 \mathrm{~cm}$ ), y una anchura media de $14 \mathrm{~m}$. En ambos puntos el sustrato estuvo compuesto de cantos, bloques y losa, junto a algunas áreas con gravas.

\section{MATERIAL Y MÉTODOS}

Se analizaron 70 gobios (de longitud total entre 30 y $136 \mathrm{~mm}$ ) capturados mediante pesca eléctrica en verano de 1996 y 1997 (19 ejemplares en el punto P1 y 51 en el punto P2). Los ejemplares fueron capturados antes del mediodía, ya que el gobio tiene un ritmo de alimentación principalmente matinal (Neveu, 1981). Tras su captura, fueron trasladados en refrigeración al laboratorio, donde se congelaron para su posterior estudio. El digestivo fue separado y conservado en formaldehido al $4 \%$. Su contenido fue vaciado y observado a la lupa binocular (x7-45). Se establecieron tres grupos de presas: materia vegetal, invertebrados terrestres (insectos) e invertebrados acuáticos. En este último grupo, siempre que fue posible se determinaron las presas hasta nivel de familia. La materia vegetal fue cuantificada visualmente en cuanto a su abundancia, anotándose también el número de estómagos en los que aparecía.

En las presas animales se determinó su abundancia A (porcentaje respecto al número total de presas) y frecuencia $\mathrm{F}$ (porcentaje de digestivos donde aparecía). Se calculó la diversidad trófica según el índice de Shannon $\left(\mathrm{H}^{\prime}=-\Sigma \mathrm{pi} \cdot \log _{2} \mathrm{pi}\right)$, donde pi es la frecuencia relativa de la presa $\mathrm{i}$. Para analizar si había diferencias en la dieta de los gobios según la edad, se comparó el contenido digestivo de 27 gobios de edad inferior o igual a dos años con el de 17 gobios de edad superior a dos años, todos ellos capturados en el punto P2 en verano de 1996. La variación de la dieta entre las clases de longitud y entre puntos de muestreo se comprobó con el test $\chi^{2}$. 
Además se analizó la selección de presas del gobio en el punto P2 comparando la composición de su dieta con los macroinvertebrados presentes en el río. Para ello se muestrearon todos los microhábitats existentes en el tramo con una manga de recolección de $35 \mathrm{~cm}$ de boca y malla de $0.1 \mathrm{~mm}$ de luz, durante un periodo de 10-15'. La muestra tomada se fijaba en el lugar de captura con formaldehido y se llevaba al laboratorio, donde se contaban y clasificaban todos los macroinvertebrados de la misma. La selección se comprobó mediante el índice S de Savage (1931) S = Ui/Di, donde Ui es el uso que el gobio hace del recurso i, y Di es la disponibilidad de ese recurso en el río, ambos datos en expresados en porcentaje. Los valores de $\mathrm{S}$ varían entre 0 e $\infty$, siendo 1 el valor correspondiente a la no selección. Se eligió este índice en lugar de otros porque permite comprobar su significación estadística comparándola con una $\chi^{2}$ de un grado de libertad (Manly et al., 1993).

Tabla 1. Composición de la dieta del gobio en el río Larraun (Navarra).(F: Frecuencia; A: Abundancia; H’: Diversidad trófica). Diet composition of gudgeon in the Larraun river (Navarra, North Spain). F: Ocurrence (frecuency of each item over total stomache contents analyzed); A: Abundance; H': Trophic diversity.

\begin{tabular}{|c|c|c|c|c|c|c|}
\hline & \multicolumn{2}{|c|}{ P1 } & \multicolumn{2}{|c|}{$\mathbf{P 2}$} & \multicolumn{2}{|c|}{ Total } \\
\hline & $\mathbf{F}$ & $\mathbf{A}$ & $\mathbf{F}$ & $\mathbf{A}$ & $\mathbf{F}$ & $\mathbf{A}$ \\
\hline \multicolumn{7}{|l|}{ Invertebrados acuáticos } \\
\hline Nematoda & & & 9.09 & 2.20 & 6.67 & 1.29 \\
\hline Hidracarina & & & 6.82 & 0.47 & 5.00 & 0.28 \\
\hline Ancylidae & 6.25 & 0.22 & 6.82 & 0.47 & 6.67 & 0.37 \\
\hline Anomopoda & 62.50 & 37.00 & 4.55 & 0.47 & 20.00 & 15.70 \\
\hline Pacifastacus & & & 11.36 & 0.79 & 8.33 & 0.46 \\
\hline Gamaridae & & & 20.45 & 2.05 & 15.00 & 1.19 \\
\hline Ostracoda & 6.25 & 0.22 & 2.27 & 0.16 & 3.33 & 0.18 \\
\hline Elmidae (Adulto) & & & 2.27 & 0.16 & 1.67 & 0.09 \\
\hline Chironomidae (Larva) & 100.00 & 53.96 & 95.45 & 69.29 & 96.67 & 62.90 \\
\hline Chironomidae (Pupa) & 50.00 & 4.19 & 11.36 & 0.94 & 21.67 & 2.30 \\
\hline Limoniidae & & & 15.91 & 1.10 & 11.67 & 0.64 \\
\hline Simulidae (Larva) & & & 2.27 & 0.16 & 1.67 & 0.09 \\
\hline Simulidae (Pupa) & & & 2.27 & 0.16 & 1.67 & 0.09 \\
\hline Baetidae & 12.50 & 0.44 & 11.36 & 0.79 & 11.67 & 0.64 \\
\hline Caenidae & & & 4.55 & 0.31 & 3.33 & 0.18 \\
\hline Heptageniidae & 6.25 & 0.22 & & & 1.67 & 0.09 \\
\hline Leuctridae & & & 2.27 & 0.16 & 1.67 & 0.09 \\
\hline Hydroptilidae & 12.50 & 0.44 & & & 3.33 & 0.18 \\
\hline Hydropsychidae & & & 9.09 & 0.94 & 6.67 & 0.55 \\
\hline Psychomyidae & 6.25 & 0.22 & 52.27 & 9.92 & 40.00 & 5.88 \\
\hline Trichoptera indet. & 6.25 & 0.22 & 4.55 & 0.31 & 5.00 & 0.28 \\
\hline Artropoda indet. & 6.25 & 0.22 & 6.82 & 0.47 & 6.67 & 0.37 \\
\hline Total & 100.00 & 97.36 & 100.00 & 91.34 & 100.00 & 93.85 \\
\hline \multicolumn{7}{|l|}{ Invertebrados terrestres } \\
\hline Coleoptera & & & 2.27 & 0.16 & 1.67 & 0.09 \\
\hline Formicidae & & & 2.27 & 0.16 & 1.67 & 0.09 \\
\hline Diptera & & & 52.27 & 5.98 & 38.33 & 3.49 \\
\hline Trichoptera & & & 18.18 & 1.42 & 13.33 & 0.83 \\
\hline Insecta indet. & 25.00 & 2.64 & 13.64 & 0.94 & 16.67 & 1.65 \\
\hline Total & 25.00 & 2.64 & 70.45 & 8.66 & 58.33 & 6.15 \\
\hline Restos vegetales & 6.25 & - & 50.00 & - & 38.33 & - \\
\hline $\mathrm{N}^{\mathrm{o}}$ digestivos & \multicolumn{2}{|c|}{16} & \multicolumn{2}{|c|}{44} & \multicolumn{2}{|c|}{60} \\
\hline $\mathrm{N}^{\circ}$ presas & \multicolumn{2}{|c|}{454} & \multicolumn{2}{|c|}{635} & \multicolumn{2}{|c|}{1089} \\
\hline $\mathrm{H}^{\prime}$ & \multicolumn{2}{|c|}{1.53} & \multicolumn{2}{|c|}{1.94} & \multicolumn{2}{|c|}{2.08} \\
\hline
\end{tabular}




\section{RESULTADOS}

De los 70 digestivos estudiados 10 de ellos se encontraron vacíos, y no se tuvieron en cuenta en análisis posteriores. Las presas contenidas en los 60 restantes fueron 1089 (Tabla 1), principalmente quironómidos, seguidos de cladóceros, invertebrados de origen terrestre (sobre todo dípteros) y tricópteros (psicómidos principalmente). En 23 digestivos se hallaron restos de materia vegetal, aunque siempre se encontró en muy bajas cantidades, y nunca apareció como único recurso trófico utilizado. Además en 18 digestivos se constató la presencia de restos de sustrato (arena y sedimentos principalmente), muchas veces coincidiendo con la presencia de materia vegetal (15 digestivos).

La composición de la dieta fue significativamente distinta entre ambos puntos de muestreo $\left(\chi^{2}=243.2 ; 5\right.$ g.1.; $\left.\mathrm{p}<0.001\right)$. En el punto P1 la dieta se compuso principalmente de quironómidos seguidos de cladóceros, mientras que en el punto P2 las presas más consumidas fueron quironómidos, seguidos de invertebrados terrestres y psicómidos, mientras que los cladóceros fueron poco consumidos (Tabla 1). Además en el punto P2 hubo mayor frecuencia en la aparición

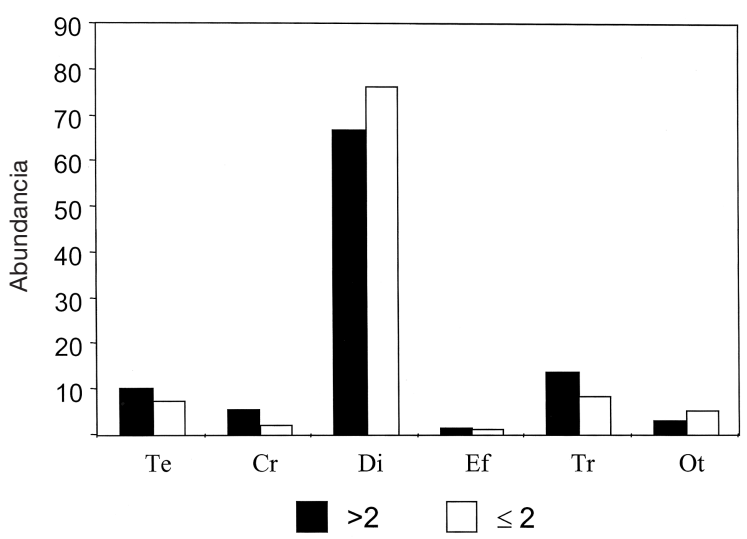

Figura 2. Composición de la dieta del gobio en el río Larraun (Navarra) según la edad. (Te: Invertebrados terrestres; Cr: Crustáceos; Di: Dípteros; Ef: Efémeras; Tr: Tricópteros; Ot: Otras presas). Food of gudgeon from the Larraun river (Navarra, North Spain) according to age. (Te: Terrestrial invertebrates; Cr: Crustacea; Di: Diptera; Ef: Ephemeroptera; Tr: Trichoptera; Ot: Other prey). de materia vegetal en el digestivo. Estos cambios hicieron que la diversidad trófica fuera mayor en el punto $\mathrm{P} 2$.

La composición de la dieta también varió significativamente con la edad $\left(\chi^{2}=14.7 ; 5\right.$ g.1.; $\mathrm{p}<0.05)$. Los gobios de mayor edad consumieron proporcionalmente menos dípteros (Fig. 2), aumentando el consumo de crustáceos, tricópteros y presas de origen terrestre. Estos cambios hicieron que la diversidad trófica se incrementara con la edad $\left(\mathrm{H}^{\prime}=1.59\right.$ para los gobios de edad $\leq 2$, y $H^{\prime}=2.17$ en los de edad superior a 2 años). Asimismo, el número medio de presas por digestivo fue significativamente superior en los gobios de edad superior a dos años $(U=146.5 ; \mathrm{p}<0.05)$, con 13.3 presas por estómago para los gobios menores y 18.1 para los mayores. Asimismo el número de categorías de presas consumidas fue mayor en los de más edad (17 para los gobios $\leq$ años y 21 en los de edad superior a dos años).

Por otra parte, los gobios seleccionaron positivamente quironómidos, limónidos y psicómidos, y negativamente ácaros, gammáridos, élmidos, efémeras y plecópteros (Tabla 2).

\section{DISCUSIÓN}

La dieta de los gobios en el río Larraun estuvo compuesta principalmente por invertebrados bentónicos, algo lógico teniendo en cuenta los hábitos de esta especie (Chappaz et al., 1988) y la posición ínfera de la boca que puede limitar el uso de presas terrestres o superficiales (Magalhães, 1993). A pesar de ello los gobios también consumieron presas de origen terrestre.

Las presas más consumidas fueron los quironómidos, algo ya apuntado en otros ríos europeos (Penczak et al., 1984; Przybylski \& Banbura, 1989, Magalhães, 1993). La materia vegetal no fue ingerida en grandes cantidades, siendo consumida de manera accidental, y sólo se encontró en estómagos en los que también estaban presentes los dípteros, algo ya apuntado por Przybylski (1988) en Polonia. El bajo consumo de materia vegetal pudo estar relacionado con la 
Tabla 2. Selección de presas por los gobios en el punto $\mathrm{P} 2$ en el río Larraun (Navarra; ns: $\mathrm{P}>0.05 ; * * \mathrm{P}<0.01$ ). El nivel de significación se obtuvo tras la corrección de Bonferroni (a/número de categorías)). Prey selection by gudgeon from P2 in Larraun river (Navarra, North Spain). ( $U_{i}=$ use; $D_{i}=$ availability; $S=$ Savage index; $\left.n s: P>0.05 ; * * P<0.01\right)$. Significance levels were obtained after applying the Bonferroni correction (a/number of categories).

\begin{tabular}{|c|c|c|c|c|c|c|c|}
\hline \multirow[b]{2}{*}{ Presas } & \multicolumn{4}{|c|}{ Edad 2} & \multicolumn{3}{|c|}{ Edad $>2$} \\
\hline & $\mathbf{D}_{\mathbf{i}}$ & $\mathbf{U}_{\mathbf{i}}$ & $\mathbf{S}$ & & $\mathbf{U}_{\mathbf{i}}$ & $\mathbf{S}$ & \\
\hline Hidracarina & 10.260 & 0.373 & 0.0364 & $* *$ & 0.725 & 0.071 & $* *$ \\
\hline Ancylidae & 0.181 & 0.000 & 0.0000 & & 1.087 & 6.004 & $* *$ \\
\hline Anomopoda & - & 0.746 & - & & - & - & \\
\hline Gammaridae & 11.285 & 1.119 & 0.0992 & $* *$ & 3.623 & 0.321 & $* *$ \\
\hline Ostracoda & 0.060 & - & 0.0000 & & 0.362 & 6.004 & \\
\hline Pacifastacus leniusculus & - & - & & & 1.812 & - & \\
\hline Elmidae & 4.406 & - & 0.0000 & $* *$ & 0.362 & 0.082 & $*$ \\
\hline Chironomidae & 27.761 & 80.224 & 2.8898 & $* *$ & 72.464 & 2.610 & $* *$ \\
\hline Limoniidae & 0.181 & 1.119 & 6.1828 & $* *$ & 1.087 & 6.004 & $* *$ \\
\hline Simuliidae & - & - & & & 0.362 & - & \\
\hline Baetidae & 5.009 & 1.119 & 0.2235 & $*$ & 0.725 & 0.145 & $*$ \\
\hline Caenidae & 24.562 & - & 0.0000 & $* *$ & 0.725 & 0.030 & $* *$ \\
\hline Leuctridae & 6.457 & 0.373 & 0.0578 & $* *$ & - & 0.000 & $* *$ \\
\hline Hydropsychidae & 0.604 & 0.373 & 0.6183 & & 1.812 & 3.002 & \\
\hline Psychomyidae & 0.181 & 9.701 & 53.5846 & $* *$ & 13.406 & 74.045 & $* *$ \\
\hline Otros & 9.053 & 4.851 & 0.5358 & & 1.449 & 0.160 & $* *$ \\
\hline
\end{tabular}

menor tasa de absorción y el escaso contenido energético de la materia vegetal (Lien, 1981), así como con la pequeña longitud del estómago del gobio que provocaría una menor eficiencia en su procesado (Magalhães, 1993). Esto sugiere que para los gobios las presas animales resultarían energéticamente más rentables que la materia vegetal (Penczak et al., 1984).

La presencia de restos de sustrato es algo ya apuntado por anteriores estudios (Penczak et al.; 1984, Przybylski, 1988), pudiendo proceder de los restos contenidos en los digestivos de los invertebrados consumidos (Kennedy \& Fitzmaurice, 1972) o ser debidos a ingestas accidentales al capturar los invertebrados que habitan el bentos.

Las diferencias encontradas en la dieta de los gobios en los dos puntos del río Larraun podrían ser un reflejo de la distinta disponibilidad y abundancia de invertebrados bentónicos en ellos, motivadas por las diferentes condiciones de hábitat. Aunque en ambos puntos los quironómidos fueron la presa principal, el consumo de pequeños crustáceos en el punto P1 fue más del doble que el hallado en el punto P2. Esto podría deberse a que el punto $\mathrm{P} 1$ era un tramo muy remansado, lo cual probablemente favoreció el consumo de este tipo de presas (Kennedy \& Fitzmaurice, 1972). El mayor consumo de presas de origen terrestre en el punto P2 pudo estar también relacionado con la diferente topografía (presencia de cortados y taludes, vegetación riparia y velocidad del agua) (Vollestad \& Andersen, 1985).

La variación de la dieta con el aumento de talla del gobio es un hecho apuntado por Kennedy \& Fitzmaurice (1972) para Irlanda. Una mayor talla hace posible que los gobios consuman presas de mayor tamaño, de forma que el espectro de presas potenciales aumenta. Esto podría permitir una diversificación de la dieta, propiciando una menor ingesta de dípteros y un mayor consumo de otros grupos como tricópteros, presas terrestres o crustáceos, lo que se traduciría en un aumento de la diversidad trófica. Todo ello podría ser una respuesta funcional a la variación en el tamaño de la boca del pez (Neveau et Thibault, 1977), aunque Easton \& Orth (1992) señalan que 
la poca habilidad natatoria de los peces menores también puede influir en un mayor consumo de presas no evasivas, tales como quironómidos. El aumento en la talla del pez con la edad también podría favorecer tener un mayor número de presas en el estómago, algo ya observado en salmónidos (Neveau et Thibault, 1977).

El consumo preferente de quironómidos, limónidos o psicómidos puede relacionarse con los hábitos bentónicos del gobio, ya que estos taxones se hallan muy asociados al sustrato. Además, al ser presas de escasa movilidad serían más fácilmente capturables (Vinyard, 1980). Por el contrario, élmidos y gammáridos podrían ser menos rentables energéticamente debido a su intensa esclerotización, lo que les llevaría a ser rechazados por los gobios, como también ha sido observado para truchas y piscardos en este mismo río (Oscoz et al., 2000 y 2001). También, y puesto que el gobio es un depredador visual, las presas de pequeño tamaño (como los ácaros) o las que se mimetizan u ocultan bajo el sustrato (algunas efémeras y plecópteros) serían más difícilmente detectadas (Ware, 1973; Rajasilta \& Vuorinen, 1983), resultando por ello menos consumidas.

\section{BIBLIOGRAFÍA}

CHAPPAZ, R., G. BRUN et G. OLIVARI. 1988. Stratégie de développement du goujon Gobio gobio (L.) dans un lac oligotrophe: la retenue de Sainte-Croix (Provence, France). C.R, Acad. Sci. Paris, 307, Serie III: 493-497.

DOADRIO, I., 2001. Atlas y libro rojo de los peces continentales de España. Mus. Nac. Cienc. Nat. (CSIC), Ministerio de Medio Ambiente, Madrid, 364 pp.

DOADRIO, I. y B. ELVIRA. 1986. Sobre la distribución de Gobio gobio (L., 1758) (Ostariophysi, Ciprinidae) en España. Doñana, Acta Vertebrata, 13: $165-166$.

EASTON, R. S. \& D. J. ORTH. 1992. Ontogenic diet shifts of age 0 smallmouth bass (Micropterus dolomeu Lacepede) in the New River, West Virginia, USA. Ecology of Freshwater Fish, 1: 8698.
KENNEDY, M. \& P. FITZMAURICE. 1972. Some aspects of the biology of gudgeon Gobio gobio (L.) in Irish waters. J. Fish Biol., 4: 425-440.

LIEN, L., 1981. Biology of the minnow Phoxinus phoxinus and its interactions with brown trout Salmo trutta in Ovre Heimdalsvatn, Norway. Holartic Ecology, 4: 191-200

LOBÓN-CERVIÁ, J., C. MONTAÑÉS \& A. DE SOSTOA. 1991. Influence of environment upon the life history of gudgeon, Gobio gobio (L.): a recent and successful colonizer of the Iberian Peninsula. J. Fish Biol., 39: 285-300.

LOBÓN-CERVIÁ. J. \& S. TORRES. 1983. On the growth and reproduction of two populations of gudgeon (Gobio gobio L.) in Central Spain. Acta Hydrobiol., 25/26: 101-115.

MAGALHÃES, M. F. 1993. Feeding of an Iberian stream cyprinid assemblage: seasonality of resource use in a highly variable environment. Oecologia, 96: 253-260.

MANLY, B., L. MCDONALD \& D. THOMAS. 1993. Resource selection by animals. Statistical design and analysis for field studies. Chapman \& Hall, Londres.

MANN, R. H. K. 1980. The growth and reproductive strategy of the gudgeon, Gobio gobio (L.), in two hard-water rivers in southern England. J. Fish Biol., 17: 163-176.

NEVEAU, A. et M. THIBAULT. 1977. Comportament alimentaire d'une population sauvage de Truite fario (Salmo trutta L.) dans un ruisseau des Pyrènèes atlantiques, Le Lissuraga. Ann. Hydrobiol., 8: 111-128.

NEVEU, A. 1981. Rythme alimentaire et relations trophiques chez l'anguille (Anguilla anguilla L.), la loche franche (Nemacheilus barbatulus L.), le vairon (Phoxinus phoxinus L.) et le goujon (Gobio gobio L.) dans des conditions naturelles. Bull. Cent. Etud. Rech. sc., Biarritz 13(4): 431-444.

OSCOZ, J., M. C. ESCALA y F. CAMPOS. 2000. La alimentación de la trucha común (Salmo trutta L. 1758) en un río de Navarra (N. España). Limnetica, 18: 29-35.

OSCOZ, J., F. CAMPOS y M. C. ESCALA. 2001. Alimentación del piscardo (Phoxinus phoxinus) en un río del Norte de España. Ecologia, 15: 285-291.

PENCZAK, T., E. KUSTO, D. KRYZANOWSKA, M. MOLINSKI y E. SUSZYCKA. 1984. Food consumption and energy transformations by fish populations in two small lowland rivers in Poland. Hydrobiologia, 108: 135-144. 
PRZYBYLSKI, M. 1988. Food of dace, bream, gudgeon and stone loach in the Widawka river. Acta Univ. Lodz., Folia Limnol., 3: 101-115.

PRZYBYLSKI, M. \& J. BANBURA. 1989. Feeding relations between the gudjeon (Gobio gobio (L.)) and the stone loach (Noemacheilus barbatulus (L.)). Acta Hydrobiol., 31(1/2): 109-119.

RAJASILTA, M. \& I. VUORINEN. 1983. A field study of prey selection in planktivorous fish larvae. Oecologia, 59: 65-68.

SAVAGE, R. E. 1931. The relation between the feeding of the herring off the cast coast of England and the plankton of the surrounding waters. Fishery
Investigation, Ministry of Agriculture, Food and Fisheries, Series 2, 12: 1-88.

VINYARD, G. L. 1980. Differential prey vulnerability and predator selectivity: the effects of evasive prey on sunfish (Lepomis) predation. Canadian Journal of Aquatic Science, 37: 2294-2299.

VOLLESTAD, L. A. \& R. ANDERSEN. 1985. Resource partitioning of various age groups of brown trout Salmo trutta in the littoral zone of Lake Selura, Norway. Arch. Hydrobiol., 105 (2): 177-185. WARE, D. M. 1973. Risk of epibenthic prey to predation by rainbow trout (Salmo gairdneri). J. Fish. Res. Board Can., 30: 787-797. 
